\title{
On-line Detection Method of Power Quality Detection Device Based on High-precision Standard Time Synchronization Technology
}

\author{
Rui Chen \\ Wuhu Institute of Technology, Anhui Province Wuhu241000, China \\ *Corresponding Author Email: ruichen1831@163.com
}

Received: April 14, 2020. Revised: August 20, 2020. 2nd Revised: October 12, 2020. Accepted: October 19, 2020. Published: October 23, 2020.

\begin{abstract}
To improve power quality and improve the operational efficiency and accuracy of industrial automation machinery, the on-line detection method of power quality detection device based on high precision standard time synchronization technology is studied. Firstly, the research situation of power quality and time synchronization technology is briefly analyzed, and the Network Time Protocol (NTP) time synchronization technology and the calculation method of basic power quality are introduced. Then, based on the high-precision standard time synchronization technology, the system scheme of the power quality detection device is designed. Finally, the system is tested, and the test results are in line with the test expectation, indicating that the system can make a particularly accurate measurement of power quality. The power quality detection system designed in this research improved the detection performance of power quality and is of great significance to the research on online detection of power quality.
\end{abstract}

Keywords - time synchronization, power quality, digital signal processor (DSP), on-line detection

\section{INTRODUCTION}

Electric energy is the embodiment of the work done by electric power. And electric power is also the most basic energy source in civil and industrial use. The invention of electricity made a great leap forward in human life. At present, people can't live without electric energy in business operation. At the same time, it is also a kind of quality product provided by the power sector for users. Now as a commodity, electrical energy has high requirements for quality. Power quality, as the name suggests, is the quality of electrical energy. Parameters that measure power quality include voltage, frequency, and waveform. It is precisely because people rely more and more on electric power that they have higher and higher requirements on power quality. And power quality can be evaluated from many parameters, such as voltage imbalance, voltage surge and so on [1-2].

At present, the clock synchronization technology adopted in the power system can't meet the high requirement due to its specific application environment and the influence of other aspects in the system. In addition, some subsystems in the power system also put forward higher requirements for the reliability of the equipment due to the harsh conditions of machinery, climate (including temperature, humidity) and dust in the substation industrial site. Therefore, in order to further improve the operation management level of the power system, meet the requirements of rapid development of the system, and improve the control ability and fault analysis ability of the system, building a perfect time synchronization network with unified technical scheme becomes a choice and direction of efforts of the power system, and the time synchronization network built also becomes an important foundation for supporting the network in the power grid [3].

Based on this, under the power quality and time synchronization technology, through the description of the Network Time Protocol (NTP) time synchronization technology and the calculation method of basic power quality, the high-precision standard time synchronization technology is applied. It completes the system scheme design of the power quality detection device. It is aimed to provide a certain reference for the further improvement of power quality and industrial automation efficiency. 


\section{LITERATURE REVIEW}

Hirschmann company proposed an IEEE 1588 synchronization component model. The hardware part includes a high-precision real-time clock and a timestamp unit to generate the timestamp, and the software part is bound to the real-time clock and hardware timestamp unit to implement the IEEE1588 protocol. Hirschmann had tested the IEEE1588 enhancement plug-in module on its Mice modular Ethernet switch, and found that its synchronization accuracy is \pm 100 nanoseconds at the maximum jitter, and the frequency distribution of the offset between the master and slave clocks is 23.95 nanoseconds in total, with an average of 4.248 nanoseconds [4-5].

Bollen pointed out that the time-domain method is the most popular and widely used method at present. This method can measure the value more accurately, and its process is not complicated and the data requirements are not strict. The specific principle of this method is to perform time-domain simulation analysis on some data in power quality. The time domain method can be mainly divided into the transient simulation of the system and the simulation of power electronics. The disadvantage of them is that the maximum frequency range determines the simulation step size, and the frequency limit of the transient state can't be determined in advance, and the numerical oscillation can be triggered during the switching and closing process of simulation speculation [6].

\section{METHODOLOGY}

\section{NTP time synchronization technology}

The purpose of NTP is to deliver a unified and standard time on the Internet. The specific implementation scheme is to specify a number of clock source websites on the network to provide users with timing services, and these websites should be able to compare with each other to improve accuracy. NTP is originally designed and implemented by professor Mills from the university of Delaware in the United States. It has been developed for nearly 40 years since it is first proposed in 1982 [7-8]. The latest accuracy of NTPv4 has reached 200 milliseconds. In the actual application process, the simplified version of NTP -SNTP (Simple Network Time Protocol) can be adopted, which has the accuracy of seconds but is relatively simple to implement [9]. In addition to estimating round-trip latency of packets over the network, the NTP protocol can independently estimate clock deviations between computers to provide accurate and robust time services in a disordered Internet environment to synchronize computer time to certain time standards [10-12]. At present, almost all timing websites are based on NTPv3, which provides time accuracy of tens of milliseconds on the Wide Area Network (WAN), sub-millisecond or higher on the Local Area Network (LAN), and higher accuracy on the dedicated time server [13].

NTP communicates in client/server mode. Assuming that the client is requesting time services from the server, the client first generates a standard NTP query packet that is sent over the network to the time server. After receiving the query packet, the server generates a standard NTP time packet according to its local time, and then sends it back to the client through the network. Both packets have a timestamp of transmission and reception, and the time offset and network delay between the client and the server are determined based on the four timestamps. The working principle of the NTP protocol is shown in Figure 1. 




Fig. 1 The working principle of the NTP protocol

\section{NTP network architecture}

The NTP protocol takes Universal Time Coordinated (UTC) as the time standard and can be implemented by different network structures according to requirements. For large communication networks, a hierarchical tree structure is generally adopted, and the time is propagated according to the level of the NTP server. The time server close to the UTC source has a higher time accuracy, and the accuracy of the time server is represented by a value called Stratum. According to the distance of each time server from the UTC time source into the different layers (Stratum), the network structure shown in Figure 2 can be used to implement the NTP service.
Specifically, the network structure in which the NTP service can be implemented includes four modules, namely, Stratum0, Stratum1, Stratum2, and Stratum3. The components of the first module correspond to GPS time, and the other modules are all composed of time servers. Through the interaction of various time servers, the NTP server propagates time among time servers with different distances, and finally achieves corresponding services.

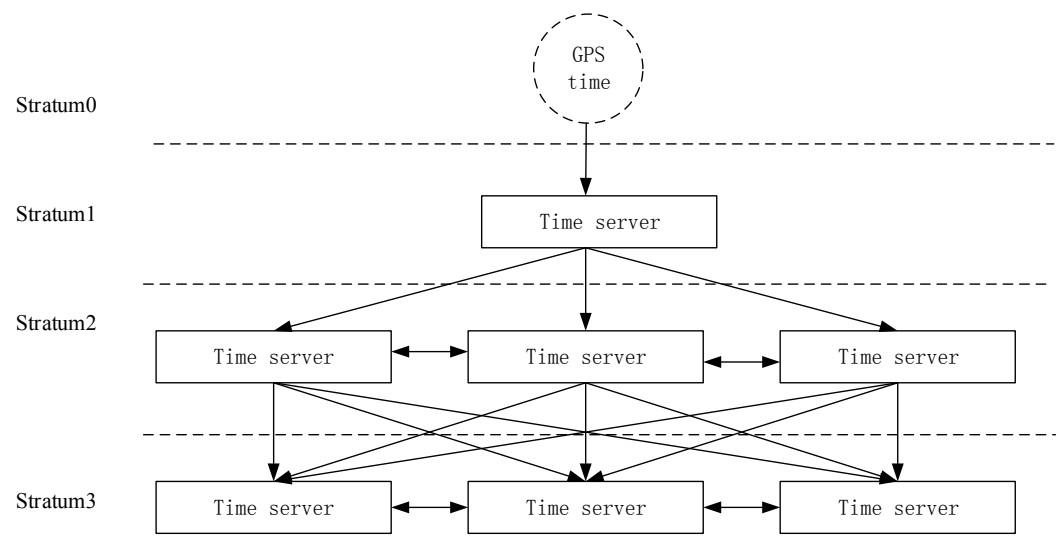

Fig. 2 Network structure of NTP 


\section{Calculation of basic power quality parameters}

There are many parameters to measure the power quality, such as voltage, current, active power, reactive power and so on. In the calculation, in the case of perfect sine wave, the formula of active power is $\mathrm{P}=\mathrm{Ul} \cos \mathrm{B}$, and when the sine wave is disturbed, the calculation formula of active power is:

$p=\frac{1}{T} \int_{0}^{T} u i d t$

The effective value of the phase voltage:

$U=\sqrt{\frac{1}{T} \int_{0}^{T} u^{2} d t}$

The effective value of the phase current:

$$
I=\sqrt{\frac{1}{T} \int_{0}^{T} i^{2} d t}
$$

For $u(t)$ and $\mathrm{i}(t)$, the discrete mode is used to obtain samples, and the discrete state sequence $\left\{u_{k}\right\}$ and $\left\{i_{k}\right\}$ is finally obtained, that is:

$U=\sqrt{\frac{1}{T} \sum_{k=0}^{N-1} u_{k}^{2} \Delta T_{k}}$

Among them, $\Delta T_{k}$ is the sampling interval of two adjacent; $\mathrm{N}$ is the number of sampling points in a cycle; $u_{k}$ is the instantaneous value represented by the voltage at time $\mathrm{k}$. If the time interval between two adjacent samples is equal, that is, $\Delta T_{k}$ is a time constant $\Delta T$, and $N=\frac{T}{\Delta T}$, then:
$U \approx \sqrt{\frac{1}{N} \sum_{k=0}^{N-1} u_{k}^{2}}$

In the same way, the current effective formula can be obtained:

$I \approx \sqrt{\frac{1}{N} \sum_{k=0}^{N-1} i_{k}^{2}}$

\section{The design of the software structure of the systam}

Software is the soul of power quality detection systems. In this software programming, the code is usually edited in a modular coding manner, the benefit of doing so is to be able to carry out necessary upgrades to the whole system in the future. The entire software writing process(z) this system is carried out in the CCS software editor. This software writing language is mainly $\mathrm{C}$ language, this kind of coding environment can complete a lot of work in the software writing process, including writing, the final debugging, and modification. This kind of writing environment is very popular at present, almogst all software practitioners write in such an environment, which has a great positive effect on compatibility and scalability of software.

The figure below shows the entire software writing process. The software writing of the system shall meet all the requirements in the hardware design and all the requirements in the system design. The system is to detect the quality of power, and the data timing must be dettected during the detection process, and this process requires hardware and software to work together. Nowadays, the device design is a combination of hardware and software. It is not the hardware that is assembled to meet the user's requirements. All of them need a simple software language to achieve, which is equivalent to its brain. The software design should satisfy all the requirements of the system, and the future expansion should be determined according to the future situation. 


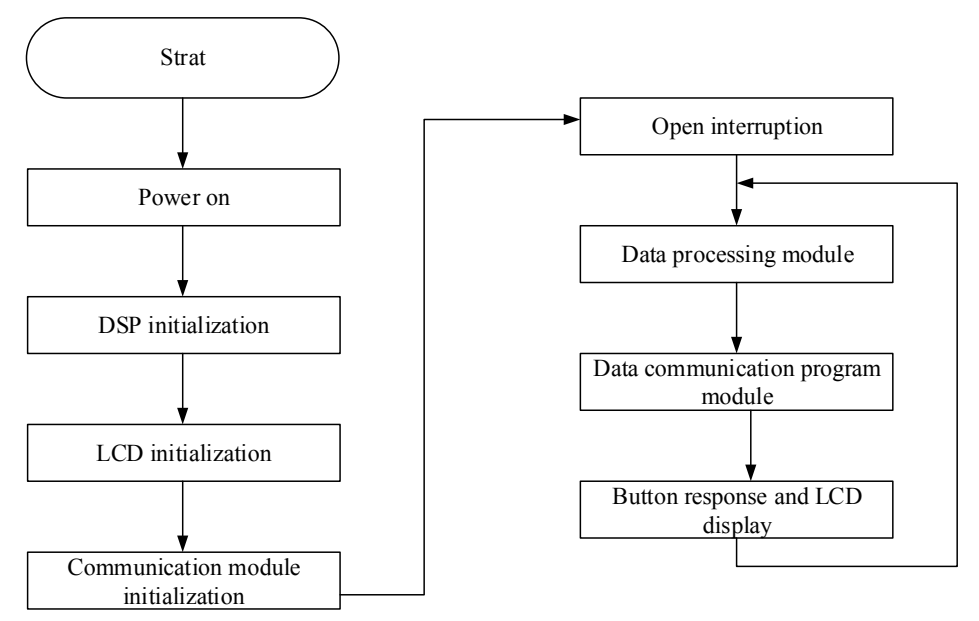

Fig. 3 Flowchart of system procedures

Figure 4 is the flow structure diagram of FFT operation. It can be concluded that when low order operation replaces high order operation, the calculation flow of the whole system is the process of analyzing data, translating data, calculating data, translating data and outputting. Although there is a process of variability here, the pressure on the core of the system is greatly reduced.

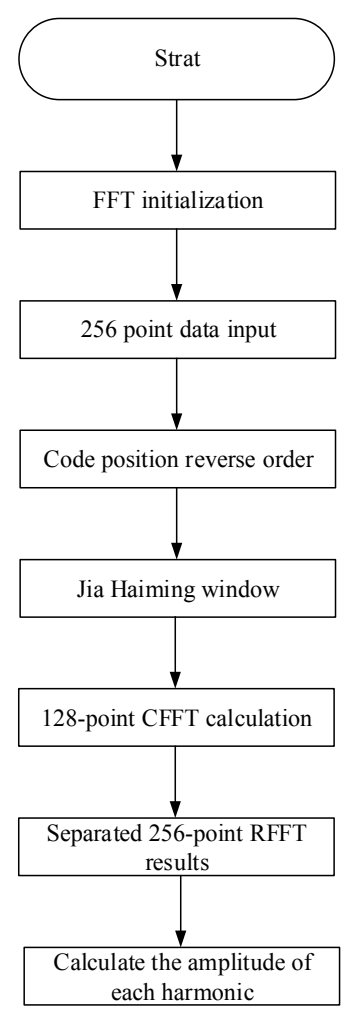

Fig. 4 FFT process

Since the clock cycle of DSP is $150 \mathrm{MHz}$, while the minimum setup time of T6963 is $100 \mathrm{~ns}$, there will be an imbalance between understanding and input. In order to balance the speed of the two, DSP is set to stagnation state during waiting 
by coding or circuit design. In the system, the unequal DSP and LCD are balanced with software coding method. The specific configuration is as follows: the establishment, activation, and hold time of Zone 2 are configured to be the largest; appropriate software delay is adopted. This ensures that the timing between DSP and LCD is normal. Figure 5 is the flow chart of LCD coding process.

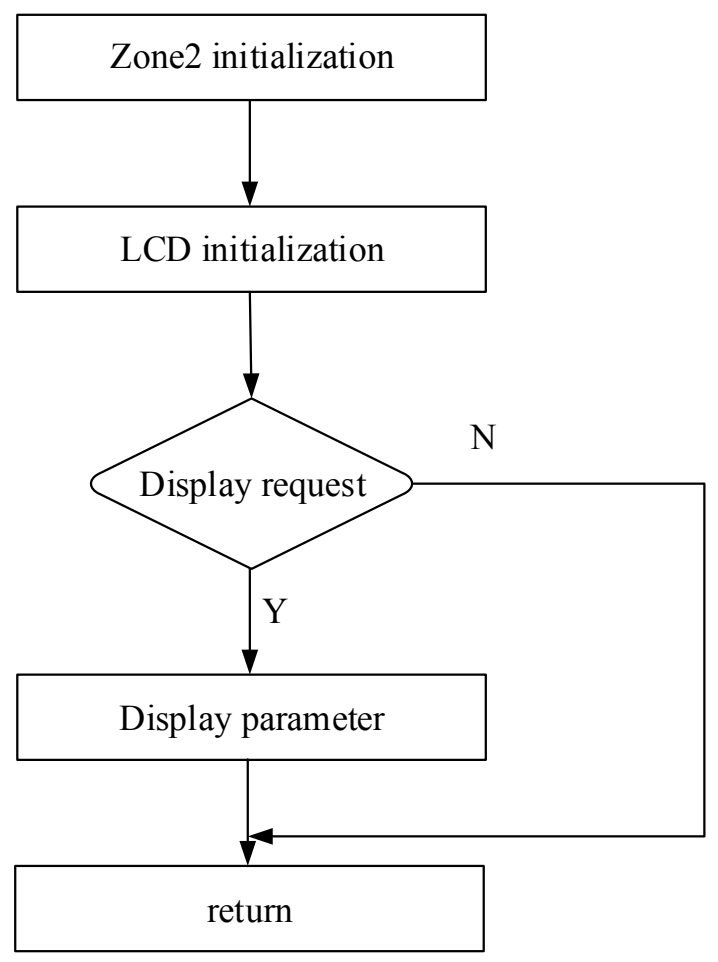

Fig. 5 LCD display flow chart

\section{RESULTS AND DISCUSSION}

\section{Data test of the system}

After the design of the power quality testing system is completed, all functions should be tested strictly according to the provisions of the national power quality standards and the performance indexes of the instrument to ensure that the testing system met the design requirements. Firstly, the measurement function is tested. The specified power signal is output by the high-precision calibrator, and the measurement result is compared with the actual input. Since the over-limit alarm function is based on the measurement function, the test is conducted at the same time. The current and voltage signals exceeding the limit are output by the calibrator, and the corresponding alarm actions of the detection system are recorded. In addition, the static simulation test is carried out on the power quality detection system to simulate various possible conditions during the online operation of the detection system, so as to verify the availability of its operation monitoring function.

Many different types of data are needed to test and analyze system data. All electrical data is converted to digital signals through the system. These digital signals can help researchers to properly detect the system. The collected standard voltage signal displayed the waveform in the CCS. The sampling frequency set by the system is $12.8 \mathrm{KHz}$, and 256 points are sampled in each power-frequency cycle.

\section{Spot test}

Basic power parameter test: frequency, voltage, current, power factor, active power and reactive power are tested 
by selecting different power factors. Through data comparison, it can be found that the voltage deviation is less than $0.5 \%$, the current deviation is less than $1 \%$, the power factor deviation is less than $1 \%$, the deviation of active power and reactive power is less than $0.5 \%$, and the frequency deviation is less than $0.02 \mathrm{~Hz}$. The system worked stably with little error. When the content rate of harmonic is less than $30 \%$, the maximum error of the system for measuring the content rate of harmonic is less than $2 \%$, and as the content rate of harmonic decreased, the measurement error also decreased.

\section{Test conclusion}

The standard of electrical error analysis is different from the general data analysis. When the value measured by such a device is analyzed for its accuracy, the standard that the accuracy can be achieved is several times or even several times that of the general measurement data. The error analysis of the measured instruments in this research is mainly from hardware circuit and software design. Many errors in hardware design are caused by voltage and current transformers, low-pass filter circuit and $\mathrm{A} / \mathrm{D}$ conversion circuit.

The errors of voltage, current and transformer are mainly caused by the excitation current and magnetic leakage voltage drop in the iron core of the transformer. The mutual inductor in this device is of good quality and high precision, and it also played a good role in linearity. Therefore, the possible error caused by this device is very small, and the so-called error would not appear in the tested system. The error of low-pass filter circuit is mainly caused by the error of operational amplifier, capacitance, resistance and so on. When the equipment started running, many factors would cause the temperature of the component to rise, which obviously had a certain effect on the amplifier.

Based on the above analysis of the causes of hardware and software errors, this study proposed the following improvements: use components with high precision and excellent linearity and components with low temperature drift; try to improve the accuracy of AID conversion and reduce the error of analog channel. Further improve the algorithm. Obviously, for some possible errors, it can be omitted by other methods.
The data of these tests showed that the system can effectively detect the power quality. The reasons of some small errors in the system are explained. In short, any sophisticated machine will have errors, but in addition to these systematic errors, this system can make a particularly accurate measurement of power quality.

\section{CONCLUSION}

In this study, firstly, the basic concepts of time synchronization and the development of time synchronization technology are outlined. As the time synchronization scheme of the power system, the time synchronization standard can greatly improve the synchronization accuracy of various power equipment within the system, ensure the accuracy of line fault location and power grid parameter calibration, improve the fault analysis ability and stability control level of power grid, and make the replacement and upgrade of time reference in system become simple and easy. Secondly, the software programming is also described in detail, including software design ideas and software programming process. Thirdly, the accuracy of the system is determined and the system is finally considered to meet the design requirements. Finally, the error is analyzed, and the method and precautions of the system anti-interference design are put forward.

The proposed algorithm can achieve effective and accurate detection of power quality. However, the on-line detection method of power quality detection device based on high-precision standard time synchronization technology is affected by many factors. There is no discussion about its practical application. It will be the direction of further deepening and expansion in the future.

\section{ACKNOWLEDGEMENTS}

The authors acknowledge The Education Department of Hunan Province Project Name: Research on High Precision Standard Time Synchronization Technology (No. 17C0134) 


\section{REFERENCES}

[1] Nantian, H., Jiajin, Q., Fuqing, L., et al. "Short-Circuit Fault Detection and Classification Using Empirical Wavelet Transform and Local Energy for Electric Transmission Line". Sensors, 2017, 17(9), 2133.

[2] Nguyen HP. "A Short Communication On Reverse Logistics Role In The Supply Chain". Information Management and Computer Science, 2019, 2(1), 10-14.

[3] Eslami, R., Sadeghi, S.H.H., Askarian-Abyaneh, H., et al. "A Novel Method for Fault Detection in Future Renewable Electric Energy Delivery and Management Microgrids, Considering Uncertainties in Network Topology". Electric Power Components and Systems, 2017, 45(10), 1118-1129.

[4] Ahmed M. A, Abdulmajed O. E, Othman I. "Stability Analysis of DC-DC Buck Converters". Acta Electronica Malaysia, 2020, 4(1), 01-06.

[5] Song, M., Lu, C., Ba, A., et al. “An Energy-Efficient Antenna Impedance Detection Using Electrical Balance for Single-Step On-Chip Tunable Matching in Wearable/Implantable Applications". IEEE Transactions on Biomedical Circuits \& Systems, 2017, 11(6), 1236-1244.

[6] Nagi, Zmarz, Y.D., Boczar, T., et al. "Detection of high-energy ionizing radiation generated by electrical discharges in oil". IEEE Transactions on Dielectrics and Electrical Insulation, 2016, 23(4), 2036-2041.

[7] Tita D, Degi A. "Individual And Organizational Impacts: Information And System Quality Influence On Attitude Towards Use And User Satisfaction Of Agency-Level Financial Application System". Acta Informatica Malaysia, 2020, 4(1), 10-18.

[8] Torres-RenterÃ-a, A., DamiÃ $¡ n-C u a l l o, \quad$ M., Mayo-Maldonado, J., et al. "Analysis of electric arc furnaces efficiency via frequency spectrum-based arc coverage detection". Ironmaking \& Steelmaking, 2017, 44(4), 255-261.
[9] Jun, X., Jing, W., Shiying, L., et al. "A Method to Simultaneously Detect the Current Sensor Fault and Estimate the State of Energy for Batteries in Electric Vehicles". Sensors, 2016, 16(8), 1328.

[10] Dilip U S, Vinay S, Shiva P, H C. "Strategic Evaluation In Optimizing The Internal Supply Chain Using Topsis: Evidence In A Coil-Winding Machine Manufacturer". Acta Mechanica Malaysia, 2020, 3(1), 1-4, DOI: 10.26480/amm.01.2020.01.04

[11] Liu, X., Chen, K., Yan, J., et al. "Optimal Energy Harvesting-based Weighed Cooperative Spectrum Sensing in Cognitive Radio Network". Mobile Networks and Applications, 2016, 21(6), 908-919.

[12] Zhenilin OU, Xie HB, Yang X, Bojia PI, De Ciel R, Xingran Zhao R.A. "A Look at Millennial Attitudes Toward Ai Utility in The Class". Information Management and Computer Science, 2019, 2(1), 07-09.

[13] Ma, C., Gao, F., He, G., et al. “A Voltage Detection Method for the Voltage Ride-Through Operation of Renewable Energy Generation Systems Under Grid Voltage Distortion Conditions". IEEE Transactions on Sustainable Energy, 2015, 6(3), 1131-1139.

\section{Creative Commons Attribution License 4.0 (Attribution 4.0 International, CC BY 4.0)}

This article is published under the terms of the Creative Commons Attribution License 4.0 https://creativecommons.org/licenses/by/4.0/deed.en_US 